radiation being of comparativeiy great wave-length. On the other hand, recent experiments by Simons (Proc. Phys. Soc. London, 37. 58 ; 1925) with ordin. ary homogeneous $\mathrm{X}$-rays have shown that in this case also a great part of the photo-electric emission consists of slow-moving $\delta$-particles.

In both cases it is possible that the process by which the large quanta of energy carried by the exciting electrons are transformed to small quanta of the photo-electrons, involves some kind of a compound photo-electric mechanism. According to Auger ( $A n n$. de Phys., 6, 183; 1926) the compound photo-electric effect should play a very important part in the soft $\mathrm{X}$-ray region.

The investigation is being continued with the object of gaining further knowledge on this point.

Stockholm, Odengatan 8,

ERIS RudBera. April 19.

\section{Correlation Coefficients and the Theory of Mental Abilities.}

SINCE the discovery by Spearman of hierarchical order in correlations between mental tests, the theory of mental ability has been actively explored. Mathematical methods have been made possible by regarding mental abilities as variables composed of certain constituent factors which are distributed unequally among individuals. The extent to which such factors of mental ability may be called into activity will presumably depend on their use in any given situation.

According to this view, we may suppose that any given test will require for its solution a number of factors of ability. If two tests are applied, more factors will as a rule come into activity, but possibly some will assist in both tests and thus produce correlation between the results. If three tests are applied, three orders of factors may be expected according as they are common to all three, or to only two out of three, or are relatively specific, i.e. applying to one out of three. If four tests are used, four orders of factors might be expected ; if five, then five orders, and so on. In any particular collection of tests, whether three or more, we regard the terms 'specific,' ' group,' and 'general' as referring to factors that are respectively (1) present only in one, (2) present in some, (3) present in all the mental processes required for the performance of those tests. In the furthest limit, when all possible variables have been considered, and provided their constituent parts could be known, the final and indivisible elements would stand out as the ultimate analysis of mental ability. However near to or remote from the actual truth this view may be, it forms an adequate method of constructing correlated variables, and is therefore of assistance in checking the interpretations that are placed upon correlation coefficients derived from experimental data.

The possibilities involved in the co-operation of constituent elements in either three or four tests have been examined by the writer, partly in conjunction with Prof. Godfrey H. Thomson of the University of Edinburgh ("The Essentials of Mental Measurement," Brown and Thomson, also Brit. Jour. Psych., 1919). In the case of any three correlated variables constructed on lines described above, the expression $r^{2}{ }_{12}+r_{13}^{2}+r^{2}{ }_{23}+2 r_{12} r_{13} r_{23}$ (where $r_{12}$ is the coefficient of correlation between the first and second variables, $r_{23}$ between the second and third, and so on) has been shown to possess special significance. Disregarding negative correlation, the limiting values for the expression are zero and five. If, however, no general factor exists between any three variables, the maximum value for this expression is unity, and any value above unity definitely indicates the presence of a general factor, while any value below unity could be produced either with or without a general factor. When the value of the expression is exactly unity we have, in this sense, a boundary condition for three variables.

In proving this boundary condition, in the first place, the following restrictions on the nature of the elements were assumed: (1) That they were normally distributed with equal standard deviations, and (2) that, when present, they acted with full effect, never with a fraction or component, as it were, of their total strength. With this second restriction removed, however, it is still true that the expression could not be greater than unity without the aid of a general factor, and any other value, unity or less than unity, can be produced with or without the aid of a general factor.

So long as the 'all or none condition' is imposed, the expression is equal to unity (general factor absent), in every case when specific factors are absent, group factors alone remaining. In the wider conditions, however, the internal construction of the group factors must conform to certain laws before the maximum value is reached. The actual details of these laws, under which the 'all or none condition' is one particular case, will, I hope, be published at an early date.

A similar inquiry has been made in the domain of four variables. Here, two boundary conditions claim attention: (1) concerning the absence of a general factor and group factors common to three out of four variables, and (2) concerning the absence of the general factor alone. For the first boundary condition we have the expression $r^{2}{ }_{12}+r^{2}{ }_{13}+r_{14}^{2}+r_{23}^{2}+r_{24}^{2} r+r_{34}^{2}-r_{12}^{2}$ $r_{34}^{2}-r_{13}^{2} r_{24}^{2}-r_{14}^{2} r_{23}^{2}+2 r_{12} r_{13} r_{23}+2 r_{23} r_{24} r_{34}+2 r_{13} r_{14} r_{34}$ $+2 r_{12} r_{14} r_{24}+2 r_{12} r_{13} r_{24} r_{34}+2 r_{13} r_{14} r_{23} r_{24}+2 r_{12} r_{14} r_{23} r_{34}$. The maximum value for this expression, without the aid of general or group factors common to three variables, is unity, and at or below unity these factors are unnecessary.

The second boundary condition requires the same expression except that the first six terms, and also the tenth to the thirteenth, all take 4 as a numerical coefficient. Such an expression possesses 16 as a maximum value without a general factor, and at or below 16 a general factor is unnecessary. Both these boundary conditions are of course true without the 'all or none' restriction.

The above studies are intended to illustrate how far inferences may be safely drawn from experimental data in the form of correlation coefficients.

University of Sheffield.

$$
\text { J. Ridley Thompson. }
$$

\section{Iron Crystals.}

IT has been found possible to grow long crystals in iron wire by making use of the allotropic transformation which occurs at about $900^{\circ} \mathrm{C}$. The method is to heat a portion of the wire between two mercury contacts by passing direct or alternating current through it and then to cause the heated portion to travel along the wire either by moving the support carrying the contacts or by moving the wire itself. The hottest part of the wire should be at $1400^{\circ} \mathrm{C}$. or higher. Under these conditions a very steep temperature gradient exists at the point where face. centred cubic $(\gamma)$ erystals, stable at high temperatures, are being replaced by body-centred cubic (a) crystals, stable at lower temperatures, and at a favourable 